\title{
On stochastic differential equations and a generalised Burgers equation
}

Dedicated to PrOfESSOR JIA-AN YAN on the Occasion of his 70th Birthday

Jiang-Lun $\mathrm{Wu}^{a}$ and Wei Yang ${ }^{b}$

a Department of Mathematics, Swansea University, Singleton Park, Swansea SA2 8PP, UK (j.l.wu@swansea.ac.uk)

${ }^{b}$ Department of Statistics, University of Warwick, Coventry, CV4 7AL, UK(Wei.yang@warwick.ac.uk)

\begin{abstract}
In this paper, we discuss a link of Itô's stochastic differential equations to nonlinear partial differential equations of Burgers type. Under certain conditions, we derive a generalised Burgers equation from a stochastic differential equation. We also give some economic interpretation of our result as well as the relevant conditions.
\end{abstract}

Mathematics Subject Classification (2000): 60H10, 35K58, 91G99.

Key Words and Phrases: Stochastic differential equations; generalised Burgers equation; porous media equation; Girsanov theorem.

\section{Introduction and the main result}

The theory of stochastic differential equations (SDEs) has been very well developed since the seminal work of the great Japanese mathematician Kiyosi Itô in the mid 1940s, cf. [12]. There are diverse applications arising from physics, biology, climate studies, engineering, systematical science to economics and finance (cf. e.g. $[6,13,15,16,19]$ and references therein. Here we would like to add two monographs $[24,7]$ which have a great influence on the younger generation of Chinese scholars). On the other hand, stochastic differential equations have profound impacts on differential geometry and partial differential equations (cf. $[5,11,14]$ and most recently [18]). The object of the present paper is to derive another (yet a complete) link from Itô's stochastic differential equations to nonlinear partial differential equations (PDEs) of Burgers type. Such assertions also hold for multidimensional 
SDEs as well as for SDEs on connected complete manifolds. In a recent joint work with Aubrey Truman and Feng-Yu Wang [20], we derive nonlinear parabolic equations of Burgers-KPZ type from multidimensional SDEs and from SDEs on connected complete manifolds, by utilising arguments different from the method given in the present paper, in such a manner that the obtained Burgers-KPZ equation characterizes the path-independance property of the density process of Girsanov transformation for the stochastic differential equation.

Let us start with a general framework for stochastic differential equations by following [11]. Let $(\Omega, \mathcal{F}, P)$ be a given complete probability space with a usual filtration $\left\{\mathcal{F}_{t}\right\}_{t \in[0, \infty)}$. We use the notation $\mathcal{A}$ to denote the collection of all $\mathcal{B}(\mathbb{R} \times[0, \infty)) / \mathcal{B}(\mathbb{R})$-measurable functions $a: \mathbb{R} \times[0, \infty) \rightarrow \mathbb{R}$.

Given $a, b \in \mathcal{A}$, we consider the following stochastic differential equation of the Markovian type for a continuous stochastic process $X=\left(X_{t}\right)_{t \in[0, \infty)}$

$$
d X_{t}=a\left(X_{t}, t\right) d t+b\left(X_{t}, t\right) d W_{t}, \quad t \geq 0
$$

where $W_{t}$ is a Brownian motion. It is well known, from e.g. [11] (cf. Theorem IV.3.1), that under the usual conditions of linear growth and locally Lipschitz, to be precise, the coefficients $a: \mathbb{R} \times[0, \infty) \rightarrow \mathbb{R}$ and $b: \mathbb{R} \times[0, \infty) \rightarrow \mathbb{R}$ satisfy linear growth and locally Lipschitz condition, $C^{2}$ with respect to the first variable, and $C^{1}$ with respect to the second variables, there exists a unique solution to equation (1.1) with given initial data $X_{0}$.

The celebrated Girsanov theorem provides a very powerful probabilistic tool to solve equation (1.1) under the name of Girsanov transformation or transformation of the drift (cf. e.g. [11]). Let $\gamma \in \mathcal{A}$ satisfy the following condition

$$
\mathbb{E}\left[\exp \left(\frac{1}{2} \int_{0}^{t}\left|\gamma\left(X_{s}, s\right)\right|^{2} d s\right)\right]<\infty, \quad \forall t>0 .
$$

Then, by Girsanov theorem (cf e.g. Theorem IV 4.1 of [11]),

$$
\exp \left(\int_{0}^{t} \gamma\left(X_{s}, s\right) d W_{s}-\frac{1}{2} \int_{0}^{t}\left|\gamma\left(X_{s}, s\right)\right|^{2} d s\right), \quad t \in[0, \infty)
$$

is an $\left\{\mathcal{F}_{t}\right\}$-martingale. Furthermore, define

$$
Q_{t}:=\exp \left(\int_{0}^{t} \gamma\left(X_{s}, s\right) d W_{s}-\frac{1}{2} \int_{0}^{t}\left|\gamma\left(X_{s}, s\right)\right|^{2} d s\right) \cdot P
$$


or equivalently in terms of the Radon-Nikodym derivative

$$
\frac{d Q_{t}}{d P}=\exp \left(\int_{0}^{t} \gamma\left(X_{s}, s\right) d W_{s}-\frac{1}{2} \int_{0}^{t}\left|\gamma\left(X_{s}, s\right)\right|^{2} d s\right)
$$

in other words, the above defined probability measure $Q_{t}$ is absolutely continuous with respect to the original probability measure $P$. Moreover, for any $T>0$,

$$
\tilde{W}_{t}:=W_{t}-\int_{0}^{t} \gamma\left(X_{s}, s\right) d s, \quad 0 \leq t \leq T
$$

is an $\left\{\mathcal{F}_{t}\right\}$-Brownian motion under the probability $Q_{T}$. Furthermore, $X_{t}$ satisfies

$$
d X_{t}=\left[a\left(X_{t}, t\right)+b\left(X_{t}, t\right) \gamma\left(X_{t}, t\right)\right] d t+b\left(X_{t}, t\right) d \tilde{W}_{t}, \quad t \geq 0 .
$$

One can then discuss comprehensively the existence and uniqueness as well as the structure of solutions to the initial value problem for equation (1.1) by appealing the above argument with suitable choice of $\gamma$. Here we want to explore such transformation to another link to partial differential equations.

Throughout the paper, we assume the diffusion coefficient $b$ satisfies that $b(x, t) \neq 0$ for any $(x, t) \in \mathbb{R} \times[0, \infty)$. Moreover, we specify the above function $\gamma$ by

$$
\gamma(x, t):=-\frac{a(x, t)}{b(x, t)}
$$

hence we further require $a$ and $b$ satisfy

$$
\mathbb{E}\left[\exp \left(\frac{1}{2} \int_{0}^{t}\left|\frac{a\left(X_{s}, s\right)}{b\left(X_{s}, s\right)}\right|^{2} d s\right)\right]<\infty, \quad \forall t>0
$$

so that the associated probability measure $Q_{t}$ is determined by

$$
\frac{d Q_{t}}{d P}=\exp \left(-\int_{0}^{t} \frac{a\left(X_{s}, s\right)}{b\left(X_{s}, s\right)} d W_{s}-\frac{1}{2} \int_{0}^{t}\left|\frac{a\left(X_{s}, s\right)}{b\left(X_{s}, s\right)}\right|^{2} d s\right) .
$$

From the abvoe expression, we can see that generally the Radon-Nikodym derivative $\frac{d Q_{t}}{d P}$ depends on the "history" of the path up to $t$, i.e., $\left\{X_{s}: 0 \leq\right.$ $s \leq t\}$. While in economics and mathematical finance studies, in particular in study of the optimal problem for the utility functions in an equilibrium 
market, it is a necessary requirement that $\frac{d Q_{t}}{d P}$ depends only on the "current" state $X_{t}$, not on the whole "history" $\left\{X_{s}: 0 \leq s \leq t\right\}$. So mathematically, one requires that the Radon-Nikodym derivative is in the form of

$$
Z\left(X_{t}, t\right)=\frac{d Q_{t}}{d P}, \quad t \in[0, \infty)
$$

for certain function $Z: \mathbb{R} \times[0, \infty) \rightarrow \mathbb{R}$. We call this the path-independent property of the density of the Girsanov transformation. In this paper, we would like to present a characterisation of this property. Our main result of this paper is formulated as follows

Theorem 1.1 Let $F: \mathbb{R} \times[0, \infty) \rightarrow \mathbb{R}$ be $C^{2}$ with respect to the first variable and $C^{1}$ with respect to the second variable. Then

$$
F\left(X_{t}, t\right)=F\left(X_{0}, 0\right) \exp \left(-\int_{0}^{t} \frac{a\left(X_{s}, s\right)}{b\left(X_{s}, s\right)} d W_{s}-\frac{1}{2} \int_{0}^{t}\left|\frac{a\left(X_{s}, s\right)}{b\left(X_{s}, s\right)}\right|^{2} d s\right)
$$

if and only if the function

$$
u(x, t):=\frac{\partial}{\partial x} \ln |F(x, t)|=\frac{a(x, t)}{b^{2}(x, t)}=-\frac{\gamma(x, t)}{b(x, t)}, \quad(x, t) \in \mathbb{R} \times[0, \infty),
$$

satisfies the following nonlinear PDE

$$
\frac{\partial}{\partial t} u=-\frac{b^{2}}{2} \frac{\partial^{2}}{\partial x^{2}} u-b\left(\frac{\partial}{\partial x} b+b u\right) \frac{\partial}{\partial x} u-b u^{2} \frac{\partial}{\partial x} b .
$$

Furthermore, if there exists a $C^{1}$-function $\Phi: \mathbb{R} \rightarrow \mathbb{R}$ such that

$$
a(x, t)=\Phi(u(x, t)), \quad(x, t) \in \mathbb{R} \times[0, \infty)
$$

then (1.2) hold if and only if the function $u(x, t)$ satisfies the following (timereversed) generalized Burgers equation

$$
\frac{\partial}{\partial t} u(x, t)=-\frac{1}{2} \frac{\partial^{2}}{\partial x^{2}} \Psi_{1}(u(x, t))-\frac{1}{2} \frac{\partial}{\partial x} \Psi_{2}(u(x, t))
$$

where

$$
\Psi_{1}(r):=\int \frac{\Phi(r)}{r} d r, \quad \Psi_{2}(r):=r \Phi(r), \quad r \in \mathbb{R} .
$$


Remark 1.2 The function $u$ defined in equality (1.3) has the following explanation. Actually, from Itô formula, one may see that the square of Brownian motion has certain contribution to the drift of the stochastic differential equation. So the composition $u\left(X_{t}, t\right)$ of the function $u$ with the process $X_{t}$ may characterize the proportion of the drift part with respect to the diffusion part in equation (1.1). Surprisingly, this function u satisfies the nonlinear PDE of Burgers type (1.5).

We would like to point out that there already exist some researches to study the behavior of the ratio between the drift and diffusion coefficients of SDEs. In the literature of mathematical finance, Hodges and Carverhill in [8] consider the stock price follows a mean-reversion type SDE

$$
\frac{d S_{t}}{S_{t}}=\left(r+\alpha\left(S_{t}, t\right) \sigma\right) d t+\sigma d W_{t}
$$

where the risk free interest rate $r$ and volatility $\sigma$ are constants, $\alpha: \mathbb{R} \times$ $[0, \infty) \rightarrow \mathbb{R}$ is a $C^{2,1}$-function. Under the assumption that the economy is chatacterised by a single representative agent maximising expected utility for wealth at terminal date $H$, they demonstrate, by using binormial tree argument in the discrete time setting and Taylor expansion, that a necessary condition for economic equilibria is that the ratio $\alpha$ follows the following Burgers equation

$$
\frac{\partial}{\partial t} \alpha(x, t)=-\frac{1}{2} \sigma^{2} \frac{\partial^{2}}{\partial x^{2}} \alpha(x, t)-\sigma \alpha(x, t) \frac{\partial}{\partial x} \alpha(x, t) .
$$

Indeed, Stein and Stein obtain in [17] the similar result for their stochastically varying volatility which is driven by an arithmetic Ornstein-Uhlenbeck process. Moreover, Hodeges and Liao in [9] consider a more general case where both the risk free interest rate $r$ and the volatility $\sigma$ are (deterministic) functions of the variables $x \in \mathbb{R}$ and $t \in[0, \infty)$, i.e., $r, \sigma: \mathbb{R} \times[0, \infty) \rightarrow \mathbb{R}$. From the following SDE

$$
\frac{d S_{t}}{S_{t}}=\left(r\left(S_{t}, t\right)+\alpha\left(S_{t}, t\right) \sigma\left(S_{t}, t\right)\right) d t+\sigma\left(S_{t}, t\right) d W_{t},
$$

they derive that the ratio (called the risk premium)

$$
v(x, t):=\frac{\alpha(x, t)}{\sigma(x, t)}
$$


satisfies the following partial differential equation

$$
\begin{aligned}
\frac{\partial}{\partial t} v(x, t)= & -\frac{1}{2} x^{2} \sigma^{2}(x, t) \frac{\partial^{2}}{\partial x^{2}} v(x, t)-x \sigma^{2}(x, t) v(x, t) \frac{\partial}{\partial x} v(x, t) \\
& -\left(x r(x, t)+x^{2} \sigma(x, t) \frac{\partial}{\partial x} \sigma(x, t)\right) \frac{\partial}{\partial x} v(x, t) \\
& -x \sigma(x, t) \frac{\partial}{\partial x} \sigma(x, t) v^{2}(x, t) \\
& -\left(x \frac{\partial}{\partial x} r(x, t)-x \sigma(x, t) \frac{\partial}{\partial x} \sigma(x, t)\right) v(x, t)+x \frac{\partial}{\partial x} r(x, t) .
\end{aligned}
$$

More recently, in $[21,22,23]$ we examine mathematically the link of SDEs of mean-reversion type arising from modeling collateralized debt obligations (CDOs) and credit risk to Burgers equation in the spirit of $[8,9]$ and we explore certain computational advantages for the associated Burgers equation.

Remark 1.3 The SDE (1.6) considered in [9] is a special case of our SDE (1.1) with

$$
a(x, t)=x[r(x, t)+\alpha(x, t) \sigma(x, t)] \quad \text { and } \quad b(x, t)=x \sigma(x, t) .
$$

In addition, the ratio $v(x, t)=\frac{\alpha(x, t)}{\sigma(x, t)}$ considered there is different from our ratio $u$ defined in (1.3) in the sense that $v$ is a partial proportion of the two coefficients of the corresponding SDE. Thus, the derived PDE (1.7) is linked to our PDE (1.5).

Our PDE (1.5) covers much more classes of specific nonlinear PDEs. Now let us give several examples to explicate this point. We assume that all conditions posed on the coefficients $a$ and $b$ above are in force.

Example 1.4 Let $a(x, t)=\sigma^{2} u(x, t)$ and $b(x, t) \equiv \sigma$, where $\sigma$ is a nonzero constant, our SDE (1.1) then becomes

$$
d X_{t}=\sigma^{2} u\left(X_{t}, t\right) d t+\sigma d W_{t}
$$

The $C^{1}$-function $\Phi$ is simply given by $\Phi(r)=\sigma^{2} r$ and the corresponding PDE (1.5) is a classical Burgers equation

$$
\frac{\partial}{\partial t} u(x, t)=-\frac{\sigma^{2}}{2} \frac{\partial^{2}}{\partial x^{2}} u(x, t)-\sigma^{2} u(x, t) \frac{\partial}{\partial x} u(x, t) .
$$

This example recovers the main result obtained in Hodges and Carverhill [8]. 
The next example shows that our PDE (1.5) can be a Porous media type partial differential equation.

Example 1.5 Let $a(x, t)=m[u(x, t)]^{m}$ and $b(x, t)=\sqrt{m}[u(x, t)]^{\frac{m-1}{2}}$, where $m \in \mathbb{N}$, our $S D E$ (1.1) then becomes

$$
d X_{t}=m\left[u\left(X_{t}, t\right)\right]^{m} d t+\sqrt{m}\left[u\left(X_{t}, t\right)\right]^{\frac{m-1}{2}} d W_{t} .
$$

The $C^{1}$-function $\Phi$ is then given by $\Phi(r)=m r^{m}$ and the corresponding PDE (1.5) is a Porous media type nonlinear PDE

$$
\frac{\partial}{\partial t} u(x, t)=-\frac{1}{2} \frac{\partial^{2}}{\partial x^{2}} u^{m}(x, t)-m \frac{\partial}{\partial x} u^{m+1}(x, t) .
$$

Our third example is to show that in the time-homogeneous case in the sense that $a$ and $b$ are functions of the variable $x \in \mathbb{R}$ only, the corresponding PDE (1.5) then determines a harmonic function.

Example 1.6 Let $a(x, t)=a(x)$ and $b(x, t)=b(x)$, our $S D E$ (1.1) then reads as follows

$$
d X_{t}=a\left(X_{t}\right) d t+b\left(X_{t}\right) d W_{t}
$$

and the corresponding PDE (1.5) is a second order elliptic equation for harmonic functions

$$
\frac{\partial^{2}}{\partial x^{2}} \Psi_{1}(u(x))+\frac{\partial}{\partial x} \Psi_{2}(u(x))=0
$$

where

$$
\Psi_{1}(r)=\int \frac{\Phi(r)}{r} d r, \quad \Psi_{2}(r)=r \Phi(r), \quad r \in \mathbb{R} .
$$

Let us finally turn to the assumptions in Theorem 1.1. We have assumed that there exists a function $F: \mathbb{R} \times[0, \infty) \rightarrow \mathbb{R}$ which is $C^{2}$ with respect to the first variable and $C^{1}$ with respect to the second variable such that

$$
F\left(X_{t}, t\right)=F\left(X_{0}, 0\right) \exp \left(-\int_{0}^{t} \frac{a\left(X_{s}, s\right)}{b\left(X_{s}, s\right)} d W_{s}-\frac{1}{2} \int_{0}^{t}\left|\frac{a\left(X_{s}, s\right)}{b\left(X_{s}, s\right)}\right|^{2} d s\right) .
$$

We would like to give some economic aspects regarding to this assumption. A conventional kind of equilibrium market can be characterized by the utility function of a representative agent (see e.g., $[10,2,3,4]$ ). Given the probability measure $P$ as an objective probability in the market model, one can 
interpret our $X_{t}$ as the wealth of the representative agent in a single stock market. If the class of utility functions is one of differentiable, increasing, and strictly concave time-separable von Neumann-Morgenstern utility functions, then the representative agent maximizes his expected utility $U$. Cox and Leland in [1] show that the path independence is necessary for expected utility maximization. By path independence, they mean that the value of a portfolio will depend only on the asset prices at that time, not on the path followed by the asset in reaching that price. Namely, the utility function $U$ depends on the state price $X_{t}$ and time $t$, for $t \geq 0$, that is, the function $U$ is of the form $U\left(X_{t}, t\right)$.

In addition, Dybvig and Ross in [4] show that, in an equilibrium market, the marginal utility in each state is proportional to a consistent state-price density function. On the other hand, in an equilibrium market, there must exist a risk neutral probability measure $Q$ which is absolutely continuous with respect to $P$. The Radon-Nikodym derivative $\frac{d Q}{d P}$ gives the state-price density [8]. Combining the above $U\left(X_{t}, t\right)$, therefore, the Radon-Nikodym derivative is also in the form of

$$
F\left(X_{t}, t\right)=F\left(X_{0}, 0\right) \frac{d Q}{d P}\left(X_{t}, t\right) .
$$

The mathematical assumption that the Radon-Nikodym derivative $F$ : $\mathbb{R} \times[0, \infty) \rightarrow \mathbb{R}$ is $C^{2}$ with respect to the first variable and $C^{1}$ with respect to the second variable can be justified by appealing Malliavin calculus and by viewing $F$ as certain conditional expectation. This is addressed in [20] with the object of multi-dimensional SDEs.

The remainder of the paper is devoted to the proof of Theorem 1.1.

\section{Proof of Theorem 1.1}

Let us start with the SDE (1.1)

$$
d X_{t}=a\left(X_{t}, t\right) d t+b\left(X_{t}, t\right) d W_{t} .
$$

Clearly by taking $\gamma(x, t)=-\frac{a(x, t)}{b(x, t)}$, from Girsanov theorem, we know that the change of measure is characterized by the Radon-Nikodym derivative

$$
\frac{d Q_{t}}{d P}=\exp \left[-\frac{1}{2} \int_{0}^{t} \frac{a^{2}\left(X_{s}, s\right)}{b^{2}\left(X_{s}, s\right)} d s-\int_{0}^{t} \frac{a\left(X_{s}, s\right)}{b\left(X_{s}, s\right)} d W_{s}\right], \quad t \geq 0 .
$$


and for any $T>0, X_{t}, 0 \leq t \leq T$, is a martingale under the probability $Q_{T}$. From our equation (1.1), we have

$$
d W_{t}=\frac{1}{b\left(X_{t}, t\right)} d X_{t}-\frac{a\left(X_{t}, t\right)}{b\left(X_{t}, t\right)} d t
$$

thus

$$
\frac{d Q_{t}}{d P}=\exp \left[-\frac{1}{2} \int_{0}^{t} \frac{a^{2}\left(X_{s}, s\right)}{b^{2}\left(X_{s}, s\right)} d s-\int_{0}^{t} \frac{a\left(X_{s}, s\right)}{b^{2}\left(X_{s}, s\right)} d X_{s}\right], \quad t \geq 0 .
$$

In order to prove the necessity, we assume that there exists a function $F: \mathbb{R} \times[0, \infty) \rightarrow \mathbb{R}$ which is $C^{2}$ with respect to the first variable and $C^{1}$ with respect to the second variable such that

$$
F\left(X_{t}, t\right)=F\left(X_{0}, 0\right) \exp \left[-\frac{1}{2} \int_{0}^{t} \frac{a^{2}\left(X_{s}, s\right)}{b^{2}\left(X_{s}, s\right)} d s-\int_{0}^{t} \frac{a\left(X_{s}, s\right)}{b^{2}\left(X_{s}, s\right)} d X_{s}\right] .
$$

Then, $Z(x, t):=\ln |F(x, t)|$ is $C^{2}$ with respect to the first variable and $C^{1}$ with respect to the second variable, and

$$
Z\left(X_{t}, t\right)=-\frac{1}{2} \int_{0}^{t} \frac{a^{2}\left(X_{s}, s\right)}{b^{2}\left(X_{s}, s\right)} d s-\int_{0}^{t} \frac{a\left(X_{s}, s\right)}{b^{2}\left(X_{s}, s\right)} d X_{s}
$$

The stochastic differential form of equality (2.1) is

$$
d Z\left(X_{t}, t\right)=-\frac{1}{2} \frac{a^{2}\left(X_{t}, t\right)}{b^{2}\left(X_{t}, t\right)} d t-\frac{a\left(X_{t}, t\right)}{b^{2}\left(X_{t}, t\right)} d X_{t}
$$

Now by viewing $Z\left(X_{t}, t\right)$ as the composition of the deterministic $C^{2,1}$ function $Z: \mathbb{R} \times[0, \infty) \rightarrow \mathbb{R}$ with the continuous semimartingale $X_{t}$, we can apply Itô Formula to $Z\left(X_{t}, t\right)$, which implies

$$
d Z\left(X_{t}, t\right)=\frac{\partial}{\partial t} Z\left(X_{t}, t\right) d t+\frac{\partial}{\partial x} Z\left(X_{t}, t\right) d X_{t}+\frac{1}{2} \frac{\partial^{2}}{\partial x^{2}} Z\left(X_{t}, t\right) b^{2}\left(X_{t}, t\right) d t .
$$

By comparing (2.2) and (2.3), the coefficients of $d t$ and $d X_{t}$ must equal respectively, thus we get

$$
\frac{\partial}{\partial x} Z\left(X_{t}, t\right)=-\frac{a\left(X_{t}, t\right)}{b^{2}\left(X_{t}, t\right)}
$$


and

$$
\frac{\partial}{\partial t} Z\left(X_{t}, t\right)+\frac{b^{2}\left(X_{t}, t\right)}{2} \frac{\partial^{2}}{\partial x^{2}} Z\left(X_{t}, t\right)=\frac{1}{2} \frac{a^{2}\left(X_{t}, t\right)}{b^{2}\left(X_{t}, t\right)} .
$$

Recall the function $u$ is defined by (1.3), we have

$$
u\left(X_{t}, t\right)=\frac{a\left(X_{t}, t\right)}{b^{2}\left(X_{t}, t\right)}
$$

Hence, (2.4) and (2.5) become respectively

$$
\frac{\partial}{\partial x} Z\left(X_{t}, t\right)=-u\left(X_{t}, t\right)
$$

and

$$
\frac{\partial}{\partial t} Z\left(X_{t}, t\right)+\frac{1}{2} \frac{a\left(X_{t}, t\right)}{u\left(X_{t}, t\right)} \frac{\partial^{2}}{\partial x^{2}} Z\left(X_{t}, t\right)=\frac{1}{2} a\left(X_{t}, t\right) u\left(X_{t}, t\right) .
$$

Since the $\operatorname{SDE}(1.1)$ is non-degenerate (as the diffusion coefficient $b \neq 0$ ), the support of $X_{t}, t \in[0, \infty)$, is the whole space $\mathbb{R}$. Hence the following two equalities

$$
\frac{\partial}{\partial x} Z(x, t)=-u(x, t)
$$

and

$$
\frac{\partial}{\partial t} Z(x, t)+\frac{1}{2} \frac{a(x, t)}{u(x, t)} \frac{\partial^{2}}{\partial x^{2}} Z(x, t)=\frac{1}{2} a(x, t) u(x, t)
$$

hold on $\mathbb{R} \times[0, \infty)$. By differentiating (2.8) with respect to the variable $x$, we get

$$
\frac{\partial^{2}}{\partial x^{2}} Z(x, t)=-\frac{\partial}{\partial x} u(x, t)
$$

then substituting (2.10) into (2.9), we have

$$
\frac{\partial}{\partial t} Z(x, t)=\frac{1}{2} \frac{a(x, t)}{u(x, t)} \frac{\partial}{\partial x} u(x, t)+\frac{1}{2} a(x, t) u(x, t) .
$$

Now, in order to eliminate $Z(x, t)$, we can differentiate $(2.11)$ with respect to $x$ and differentiate (2.8) with respect to $t$, respectively,

$$
\frac{\partial^{2}}{\partial x \partial t} Z(x, t)=-\frac{\partial}{\partial t} u(x, t)
$$


and

$$
\begin{aligned}
\frac{\partial^{2}}{\partial t \partial x} Z(x, t)= & \frac{1}{2} \frac{a(x, t)}{u(x, t)} \frac{\partial^{2}}{\partial x^{2}} u(x, t)+\frac{1}{2} \frac{\partial}{\partial x} u(x, t) \frac{1}{u(x, t)} \frac{\partial}{\partial x} a(x, t) \\
& -\frac{1}{2} \frac{\partial}{\partial x} u(x, t) \frac{a(x, t)}{u^{2}(x, t)} \frac{\partial}{\partial x} u(x, t) \\
& +\frac{1}{2} a(x, t) \frac{\partial}{\partial x} u(x, t)+\frac{1}{2} \frac{\partial}{\partial x} a(x, t) u(x, t) .
\end{aligned}
$$

Equating (2.12) and (2.13), we get

$$
\begin{aligned}
\frac{\partial}{\partial t} u(x, t)= & -\frac{1}{2} \frac{a(x, t)}{u(x, t)} \frac{\partial^{2}}{\partial x^{2}} u(x, t)+\frac{1}{2} \frac{a(x, t)}{u^{2}(x, t)}\left(\frac{\partial}{\partial x} u(x, t)\right)^{2} \\
& -\frac{1}{2}\left(\frac{1}{u(x, t)} \frac{\partial}{\partial x} a(x, t)+a(x, t)\right) \frac{\partial}{\partial x} u(x, t) \\
& -\frac{1}{2} \frac{\partial}{\partial x} a(x, t) u(x, t)
\end{aligned}
$$

which shows that equation (1.4) holds. Furthermore, from our assumption that there exists $C^{1}$-function $\Phi: \mathbb{R} \rightarrow \mathbb{R}$ such that $a(x, t)=\Phi(u(x, t))$ holds for $x \in \mathbb{R}$ and $t \geq 0$, the above equality (2.14) can be reduced to

$$
\begin{aligned}
\frac{\partial}{\partial t} u(x, t)= & -\frac{1}{2} \frac{\Phi(u(x, t))}{u(x, t)} \frac{\partial^{2}}{\partial x^{2}} u(x, t) \\
& -\frac{1}{2}\left[\frac{\Phi^{\prime}(u(x, t))}{u(x, t)}-\frac{\Phi(u(x, t))}{u^{2}(x, t)}\right]\left(\frac{\partial}{\partial x} u(x, t)\right)^{2} \\
& -\frac{1}{2}\left[\Phi(u(x, t))+u(x, t) \Phi^{\prime}(u(x, t))\right] \frac{\partial}{\partial x} u(x, t) .
\end{aligned}
$$

Next, we define

$$
\Psi_{1}(r):=\int \frac{\Phi(r)}{r} d r, \quad r \in \mathbb{R}
$$

a primitive of the function $\frac{\Phi(r)}{r}$, and

$$
\Psi_{2}(r):=r \Phi(r), \quad r \in \mathbb{R} .
$$

Then, one can easily derive

$$
\begin{aligned}
\frac{\partial^{2}}{\partial x^{2}} \Psi_{1}(u(x, t))= & \frac{\Phi(u(x, t))}{u(x, t)} \frac{\partial^{2}}{\partial x^{2}} u(x, t) \\
& {\left[\frac{\Phi^{\prime}(u(x, t))}{u(x, t)}-\frac{\Phi(u(x, t))}{u^{2}(x, t)}\right]\left(\frac{\partial}{\partial x} u(x, t)\right)^{2} }
\end{aligned}
$$


and

$$
\frac{\partial}{\partial x} \Psi(u(x, t))=\left[\Phi(u(x, t))+u(x, t) \Phi^{\prime}(u(x, t))\right] \frac{\partial}{\partial x} u(x, t) .
$$

Finally, we obtain a generalized Burgers equation

$$
\frac{\partial}{\partial t} u(x, t)=-\frac{1}{2} \frac{\partial^{2}}{\partial x^{2}} \Psi_{1}(u(x, t))-\frac{1}{2} \frac{\partial}{\partial x} \Psi_{2}(u(x, t))
$$

This ends the proof of the necessity.

Finally, let us turn to the proof of the sufficiency. We assume that there exists a function $u: \mathbb{R} \times[0, \infty) \rightarrow \mathbb{R}$ which is $C^{2}$ with respect to the first variable and $C^{1}$ with respect to the second variable which solves equation (1.4) or equation (1.5) for certain suitably chosen $\Phi$. We then specify the coefficients $a$ and $b$ of SDE (1.1) by the following

$$
a(x, t)=b^{2}(x, t) u(x, t), \quad(x, t) \in \mathbb{R} \times[0, \infty) .
$$

Now combining the above equality and (1.4) with equality (2.3), we derive (2.2) which clearly implies equality (1.3). We are done. Q.E.D.

Acknowledgment: The authors would like to thank Feng-Yu Wang for fruitful discussions, especially for the suggestion on introducing the functions $\Phi(r), \Psi_{1}(r)$ and $\Psi_{2}(r)$.

\section{References}

[1] J. C. Cox and H. E. Leland, On dynamic investment strategies. Proceedings of the Seminar on the Analysis of Security Prices, Center for research in security prices, University of Chicago, 1982.

[2] P. H. Dybvig, Inefficient dynamic portfolio in security prices or how to throw away a million dollars. Review of Financial studies, 1 (1988)(1), $67-88$.

[3] P. H. Dybvig, Distributional analysis of portfolio choice. Journal of Business, 61 (1988)(3), 369-393.

[4] P. H. Dybvig and S. A. Ross, Arbitrage, state prices and portfolio theory. Handbook of the Economics of Finance. Ed.: G.M. Constantinides, M. Harris and R. M. Stulz, Chapter 10, 1 (2003), Part 2, 605-637. 
[5] D. K. Elworthy, Stochastic Differential Equations on Manifolds. London Mathematical Society Lecture Note Series, 70, Cambridge University Press, Cambridge-New York, 1982.

[6] I. I. Gikhman and A. V. Skorohod, Stochastic Differential Equations. Grundlehren der mathematischen Wissenschaften, 218, Springer-Verlag, Berlin,Heidelberg, 1972. Berlin, 1972.

[7] S. W. He, J. G. Wang, and J. A. Yan, Semimartingale Theory and Stochastic Calculus. Science Press, Beijing; and CRC Press, Boca Raton, FL, 1992.

[8] S. Hodges and A. Carverhill, Quasi mean reversion in an efficient stock market: the characterisation of Economic equilibria which support Black-Scholes Option pricing. The Economic Journal 103 (1993), 395-405.

[9] S. Hodges S. and C. H. Liao, Equilibrium price processes, mean reversion and consumption smoothing. Working paper, 2004.

[10] C. F. Huang and R. H. Litzenberger, Foundations for Financial Economics. North-Holland, Amsterdam, 1988.

[11] N. Ikeda and S Watanabe, Stochastic Differential Equations and Diffusion Processes. North-Holland and Kodansha Ltd., Amsterdam and Tokyo, 1989.

[12] K. Itô, On stochastic differential equations. Mem. Amer. Math. Soc. 4 (1951).

[13] H. Kunita, Stochastic Flows and Stochastic Differential Equations. Cambridge University Press, Cambridge, 1990.

[14] P. Malliavin, Stochastic Analysis. Grundlehren der mathematischen Wissenschaften, 313, Springer-Verlag, Berlin,Heidelberg, 1997.

[15] P. Malliavin and A. Thalmaier, Stochastic Calculus of Variations in Mathematical Finance. Springer Finance. Springer-Verlag, Berlin, 2006.

[16] B. Øksendal, Stochastic Differential Equations. An Introduction with Applications. Sixth edition. Universitext. Springer-Verlag, Berlin, 2003. 
[17] E. M. Stein and J. C. Stein, Stock price distributions with stochastic volatility: an analytic approach. The Review of Financial Studies 4 (1991)(4), 727-752.

[18] D. W. Stroock, Partial Differential Equations for Probabilists. Cambridge Studies in Advanced Mathematics, 112. Cambridge University Press, Cambridge, 2008.

[19] D. W. Stroock and S. R. S. Varadhan, Multidimensional Diffusion Processes. Grundlehren der mathematischen Wissenschaften, 233, SpringerVerlag, Berlin,Heidelberg, 1979 and 2006.

[20] A. Truman, F.-Y. Wang, J.-L. Wu and W. Yang, A link of stochastic differential equations to Burgers-KPZ type nonlinear parabolic equations via Girsanov transformation. Submitted for publication.

[21] J.-L. Wu and W. Yang, Pricing CDO tranches in an intensity based model with the mean reversion approach. Mathematical and Computer Modelling 52 (2010) (no. 5-6), 814-825.

[22] J.-L. Wu and W. Yang, On the mechanism of CDOs behind the current financial crisis and mathematical modeling with Lévy distributions. Intelligent Information Management 2 (2010), 149-158.

[23] J.-L. Wu and W. Yang, Valuation of credit risk: the mean-reversion approach. Submitted for publication.

[24] J. A. Yan, An Introduction to Martingales and Stochastic Integrals (in Chinese). Shanghai Sci. and Tech. Publ. House, Shanghai, 1981. 